\title{
Polarimetric Parameters of Scattered Radiation in the Magnetized Plasma
}

\author{
George Jandieri $^{1}$, Akira Ishimaru², Jaromir Pistora ${ }^{1}$, Michal Lesnak ${ }^{1}$ \\ ${ }^{1}$ Nanotechnology Centre, VSB-Technical University of Ostrava, Ostrava-Poruba, Czech Republic \\ ${ }^{2}$ Department of Electrical Engineering, University of Washington, FT-10 Seattle, Washington, USA \\ *corresponding author, E-mail: georgejandieri7@gmail.com
}

\begin{abstract}
Second order statistical moments of scattered electromagnetic waves in the turbulent magnetized plasma slab with electron density fluctuations are calculated applying the modify stochastic smooth perturbation theory and the boundary conditions. The obtained results are valid for arbitrary correlation function of electron density fluctuations. Stokes parameters are analyzed both analytically and numerically. The theory predicts that depolarization effect caused by second Stokes parameter may be important in scintillation effects. Numerical calculations are carried out for new spectral function of electron density fluctuations containing both anisotropic Gaussian and power-law spectral functions using the experimental data. Polarimetric parameters are calculated for different anisotropy factor and inclination angle of elongated small-scale irregularities with respect to the magnetic lines of forces. The relationship between the scintillations and the polarimetric parameters is important.
\end{abstract}

\section{Introduction}

At the present time the features of electromagnetic waves propagation in random media is well studied [1]. The problem of depolarization of electromagnetic waves in a turbulent media has attracted considerable attention. Electron density fluctuations undergo significant influence on radio waves propagation in the turbulent magnetized plasma. Statistical moments of small-amplitude electromagnetic waves scattered by turbulent anisotropic plasma slab is important in many practical applications associated with both natural and laboratory plasmas. Angular power spectrum, scintillation effects and the angle-of-arrival of scattered electromagnetic waves by turbulent anisotropic magnetized ionospheric plasma slab for both power-law and anisotropic Gaussian correlation functions of electron density fluctuations were investigated analytically and numerically in [2-4].

The problem of depolarization of electromagnetic waves in a turbulent medium attracts considerable attention. It is known that polarized characteristics of a space radio emission caused by refraction and scattering on both density irregularities of space plasma and magnetic field bearing important information on physical conditions of a source and ionospheric plasma parameters over the path of wave propagation [5]. Particularly, radio wave depolarization effect in cosmic plasma caused by the vortex medium motion has been discussed in [6]. Variances of scattered ordinary and extraordinary waves, root-mean-square deviation of the Faraday angle by inhomogeneous magnetized plasma slab have been calculated in $[7,8]$. It was shown that isolines of the normalized Faraday angle nonlinearly depends on the inclination angle of elongated plasma irregularities and increases in proportion to the anisotropy factor.

Depolarization of electromagnetic radiation in a cold plasma with random magnetic inhomogeneities

applying stochastic perturbation theory has been considered in $[9,10]$. Simple expressions for the Stokes parameters as a function of distance and one physical parameter characterizing the interstellar plasma has

been obtained which is valid in the parabolic approximation for plane wave propagation.

The purpose of the present paper is to calculate Stokes parameters as a function of a physical parameters characterizing anisotropic irregularities of magnetized turbulent plasma and the transmission distance. Second order statistical moments of scattered radio wave have been obtained using modify stochastic smooth perturbation method taking into account the boundary conditions. Application of Stokes parameters allows to define polarization characteristics of scattered waves with a big accuracy in inhomogeneous plasma. Numerical calculations are carried out for the spectral correlation function of electron density fluctuations containing both anisotropic Gaussian and power-law correlation functions using the experimental data. The results are valid for near and far zones with respect to plasma slab boundaries.

\section{Formulation}

The electric field in the collisionless plasma satisfies the wave equation [11]:

$$
\begin{aligned}
& \nabla \times \nabla \times \mathbf{E}-\Delta \mathbf{E}-k_{0}^{2} \mathbf{E}=-\frac{v k_{0}^{2}}{1-u}\left\{\mathbf{E}-i\left[\mathbf{E} \cdot \frac{\boldsymbol{\Omega}_{\mathbf{e}}}{\omega}\right]-\right. \\
& \left.-\left(\mathbf{E} \cdot \frac{\boldsymbol{\Omega}_{\mathbf{e}}}{\omega}\right) \frac{\boldsymbol{\Omega}_{\mathbf{e}}}{\omega}\right\},
\end{aligned}
$$

where: $\Omega_{e}=e H_{0} / m c$ is the electron gyrofrequency, $e$ and $m$ are the charge and mass of an electron, $H_{0}$ is the steady magnetic field $c$ is a light speed in the free space, $N(\mathbf{r})$ is 
the electron density in the magnetized plasma, $\Delta$ is the Laplacian, $k_{0}=\omega / c, u=\Omega_{e}^{2} / \omega^{2}$ and $\mathrm{v}(\mathbf{r})=\omega_{p}^{2}(\mathbf{r}) / \omega^{2}$ are magneto-ionic parameters, $\omega_{p}(\mathbf{r})=\left(4 \pi N(\mathbf{r}) e^{2} / m\right)^{1 / 2}$ is the plasma frequency. The second rank permittivity tensor in the homogeneous medium is:

$$
\varepsilon_{i j}=\left(1-\frac{\mathrm{v}}{1-u}\right) \delta_{i j}-\frac{\mathrm{v}}{1-u}\left(i \sqrt{u} \varepsilon_{i j k} m_{k}+u m_{i} m_{j}\right),
$$

where: $\delta_{i j}$ is the Kronecker symbol, $\varepsilon_{i j k}$ is the asymmetric tensor of the third rank, $\mathbf{m}$ is the unite vector along the external magnetic field lying in the main yz-plane. Components of the Cartesian second rank permittivity tensor of the collisionless magnetized plasma are [12]:

$$
\begin{gathered}
\varepsilon_{x x}=1-Y, \quad \varepsilon_{y y}=1-Y\left(1-u_{T}\right), \quad \varepsilon_{z z}=1-Y\left(1-u_{L}\right) \\
\varepsilon_{x y}=-\varepsilon_{y x}=i Y \sqrt{u_{L}} \equiv i \tilde{\varepsilon}_{x y}, \quad \varepsilon_{y z}=\varepsilon_{z y}=Y \sqrt{u_{L} u_{T}} \\
\varepsilon_{x z}=-\varepsilon_{z x}=-i Y \sqrt{u_{T}} \equiv-i \tilde{\varepsilon}_{x z}
\end{gathered}
$$

where: $Y=\mathrm{v} /(1-u), u_{T}=u \sin ^{2} \alpha, u_{L}=u \cos ^{2} \alpha, \alpha$ is the angle between vector $\mathbf{H}_{\mathbf{0}}$ and z-axis. The distance $L$ traveling by wave in the ionospheric plasma satisfies the condition $L / k l^{2}<<1$ ( $l$ is the characteristic spatial scale of plasma irregularities).

In the homogeneous plasma the electric field satisfies the equation:

$$
\nabla \times \nabla \times<\mathbf{E}>_{i}-k_{0}^{2} \sum_{i=1}^{3} \varepsilon_{i j}<\mathbf{E}>_{j}=0 .
$$

At oblique incidence wave:

$k_{x}=k_{0} N_{*} \sin \theta \sin \varphi \equiv k \alpha_{1}, k_{y}=k_{0} N_{*} \sin \theta \cos \varphi \equiv k \alpha_{2}$,

$$
\begin{gathered}
k_{z}=k_{0} N_{*} \cos \theta \equiv k \alpha_{3}, \\
N_{* j}^{2}=1-2 \mathrm{v}(1-\mathrm{v})\left[2(1-\mathrm{v})-u \sin ^{2} \alpha \mp\right. \\
\left.\mp \sqrt{u^{2} \sin ^{4} \alpha+4 u(1-\mathrm{v})^{2} \cos ^{2} \alpha}\right]^{-1},
\end{gathered}
$$

where: $\theta$ is the angle between wave vector $\mathbf{k}$ and $z$-axis, $\varphi$ is the angle between projection of the vector $\mathbf{k}$ on the $x y$-plane and y-axis; $N_{*}^{2}$ is the square of the refractive index of two normal plane waves (ordinary and extraordinary) propagating in a homogeneous anisotropic magnetized plasma; minus sign and index $j=1$ correspond to the extraordinary wave, plus sign and index $j=2-$ to the ordinary wave.

Substituting (5) into equation (4) determinant is equal to:

$$
\Delta(x)=A_{1} x^{4}+A_{2} x^{2}+A_{3}=0,
$$

where: $A_{1}=\left(1-\alpha_{2}^{2}-\alpha_{3}^{2}\right) \varepsilon_{x x}+\left(1-\alpha_{1}^{2}-\alpha_{3}^{2}\right) \varepsilon_{y y}+$ $+\left(1-\alpha_{1}^{2}-\alpha_{2}^{2}\right) \varepsilon_{z z}+2 \alpha_{2} \alpha_{3} \varepsilon_{y z}, A_{2}=\delta_{1}\left(\varepsilon_{y y} \varepsilon_{z z}-\varepsilon_{y z}^{2}\right)+\delta_{2}$

$\left(\varepsilon_{x x} \varepsilon_{x z}+\varepsilon_{x z}^{2}\right)+\delta_{3}\left(\varepsilon_{x x} \varepsilon_{y y}+\varepsilon_{x y}^{2}\right)-2 \alpha_{2} \alpha_{3}\left(\varepsilon_{x y} \varepsilon_{x z}+\varepsilon_{x x} \varepsilon_{y z}\right)$,

$A_{3}=\varepsilon_{x x} \varepsilon_{y y} \varepsilon_{z z}-2 \varepsilon_{x y} \varepsilon_{x z} \varepsilon_{y z}+\varepsilon_{y y} \varepsilon_{x z}^{2}-\varepsilon_{x x} \varepsilon_{y z}^{2}+\varepsilon_{z z} \varepsilon_{x y}^{2}$,

$\delta_{1}=\alpha_{1}^{2}-1, \delta_{2}=\alpha_{2}^{2}-1, \delta_{3}=\alpha_{3}^{2}-1, x=k / k_{0}$.

If the angle $\varphi=0$ (vector $\mathbf{k}$ is located in the $y z$-plane) we obtain the biquadratic equation:

$x^{4}\left[\varepsilon_{z z} \cos ^{2} \theta+\left(\varepsilon_{y y}+\varepsilon_{x x} \cos ^{2} \theta\right) \sin ^{2} \theta-\varepsilon_{x x} \sin ^{2} \theta \cos ^{2} \theta+\right.$

$\left.+2 \varepsilon_{y z} \sin \theta \cos \theta\right]-x^{2}\left[\left(\varepsilon_{y y}+\varepsilon_{x x} \cos ^{2} \theta\right) \varepsilon_{z z}+\right.$

$+\varepsilon_{x x} \varepsilon_{y y} \sin ^{2} \theta+2 \tilde{\varepsilon}_{x y} \tilde{\varepsilon}_{x z} \sin \theta \cos \theta-\tilde{\varepsilon}_{x z}^{2} \cos ^{2} \theta+$

$\left.+2 \varepsilon_{x x} \varepsilon_{y z} \sin \theta \cos \theta-\varepsilon_{y z}^{2}-\tilde{\varepsilon}_{x y}^{2} \sin ^{2} \theta\right]+\left(\varepsilon_{x x} \varepsilon_{y y} \varepsilon_{z z}-\right.$

$\left.-2 \tilde{\varepsilon}_{x y} \tilde{\varepsilon}_{x z} \varepsilon_{y z}-\tilde{\varepsilon}_{x z}^{2} \varepsilon_{y y}-\varepsilon_{x x} \varepsilon_{y z}^{2}-\tilde{\varepsilon}_{x y}^{2} \varepsilon_{z z}\right)=0$.

In anisotropic homogeneous medium, for propagation in a small cone about z-axis, there exist two characteristic waves:

$\left(\frac{k_{z I}}{k_{0}}\right)^{2}=1-\mathrm{v}\left\{1-\left[u_{T} / 2(1-\mathrm{v})\right]+\right.$

$\left.+\left[u_{L}+u_{T}^{2} / 4(1-\mathrm{v})^{2}\right]^{1 / 2}\right\}^{-1 / 2}$

$\left(\frac{k_{z I I}}{k_{0}}\right)^{2}=1-\mathrm{v}\left\{1-\left[u_{T} / 2(1-\mathrm{v})\right]-\right.$

$\left.-\left[u_{L}+u_{T}^{2} / 4(1-\mathrm{v})^{2}\right]^{1 / 2}\right\}^{-1 / 2}$,

where: $k_{z I}$ and $k_{z I I}$ are the propagation constants for the ordinary and extraordinary waves, respectively. For an incident $3 \mathrm{MHZ}$ electromagnetic wave the polarization plane is clockwise as $k_{\mathbf{I}}>k_{\mathbf{I I}}$ and the angle of the Faraday rotation $\theta_{F}=0.23 k_{0} L$ is positive.

In the quasi-longitudinal approximation $\left(\theta=0^{0}\right)$ we obtain:

$\left(\frac{k_{z I}}{k_{0}}\right)^{2}=1-\mathrm{v}+\mathrm{v} \sqrt{u} \cos \theta$,

$\left(\frac{k_{z I I}}{k_{0}}\right)^{2}=1-\mathrm{v}-\mathrm{v} \sqrt{u} \cos \theta$.

rotation of the polarization plane is clockwise, $k_{\mathbf{I}}>k_{\mathbf{I I}}$ and in this case Faraday angle decreases $\theta_{F}=0.075 k_{0} L$. Now we consider wave propagation through a medium containing random irregularities. 


\section{Statistical characteristics of scattered waves}

Each parameter in equation (1) we submit as sum of the constant mean and fluctuating terms:

$$
\mathbf{E}=<\mathbf{E}>+\mathbf{e}(\mathbf{r}), \quad \mathbf{H}_{\mathbf{0}}=<\mathbf{H}_{\mathbf{0}}>, N=<N>+n(\mathbf{r}) .
$$

Second components are random functions of position. The angular brackets indicate the statistical average. Substituting (11) into equation (1) using the perturbation method we obtain set of stochastic differential equation for a scattered electric field:

$$
\left(\frac{\partial^{2}}{\partial x_{i} \partial x_{j}}-\Delta \delta_{i j}-k_{0}^{2} \varepsilon_{i j}\right) e_{j}=j_{i},
$$

where: $\mathbf{j}=-k_{0}^{2} Y_{0}\left\{<\mathbf{E}>-i \sqrt{u_{0}}[<\mathbf{E}>\mathbf{m}]-u_{0}\right.$ $(<\mathbf{E}>\cdot \mathbf{m}) \mathbf{m}\}$, is the current density, $Y_{0}=\mathrm{v}_{0} /\left(1-u_{0}\right)$.

Let an incident wave propagates along the $\mathrm{Z}$-axis and the external magnetic field is located in the $y z$-plane $\left(\mathbf{k}_{\mathbf{0}} \| Z\right.$, $\mathbf{m} \in y z$ ). Applying the Fourier integrals to the equation (12) [11] we obtain:

$$
\begin{aligned}
& \mathbf{e}(\mathbf{r})=\int_{-\infty}^{\infty} d k_{x} \int_{-\infty}^{\infty} d k_{y} \tilde{\mathbf{e}}\left(k_{x}, k_{y}, z\right) \exp \left[i\left(k_{x} x+k_{y} y\right)\right] \\
& \mathbf{j}(\mathbf{r})=\int_{-\infty}^{\infty} d k_{x} \int_{-\infty}^{\infty} d k_{y} \tilde{\mathbf{g}}\left(k_{x}, k_{y}, z\right) \exp \left[i\left(k_{x} x+k_{y} y\right)\right] .
\end{aligned}
$$

The set of second order differential equations for a scattered electric field is:

$$
\begin{aligned}
& \frac{\partial^{2} \tilde{e}_{x}}{\partial z^{2}}-i k_{x} \frac{\partial \tilde{e}_{z}}{\partial z}+\left(k_{0}^{2} \varepsilon_{x x}-k_{y}^{2}\right) \tilde{e}_{x}+\left(k_{0}^{2} \varepsilon_{x y}+k_{x} k_{y}\right) \tilde{e}_{y}+ \\
& +k_{0}^{2} \varepsilon_{x z} \tilde{e}_{z}=-k_{0}^{2} g_{x} \\
& \frac{\partial^{2} \tilde{e}_{y}}{\partial z^{2}}-i k_{y} \frac{\partial \tilde{e}_{z}}{\partial z}+\left(k_{0}^{2} \varepsilon_{y x}+k_{x} k_{y}\right) \tilde{e}_{x}+\left(k_{0}^{2} \varepsilon_{y y}-k_{x}^{2}\right) \tilde{e}_{y}+ \\
& +k_{0}^{2} \varepsilon_{y z} \tilde{e}_{z}=-k_{0}^{2} g_{y} \\
& i k_{x} \frac{\partial^{2} \tilde{e}_{x}}{\partial z^{2}}+i k_{y} \frac{\partial \tilde{e}_{y}}{\partial z}-k_{0}^{2} \varepsilon_{z x} \tilde{e}_{x}-k_{0}^{2} \varepsilon_{z y} \tilde{e}_{y}+ \\
& +\left(k_{x}^{2}+k_{y}^{2}-k_{0}^{2} \varepsilon_{z z}\right) \tilde{e}_{z}=k_{0}^{2} g_{z} \cdot
\end{aligned}
$$

Rewrite these equations as:

$$
\begin{gathered}
\tilde{e}_{x}^{\prime \prime}+a_{1} \tilde{e}_{z}^{\prime}+b_{1} \tilde{e}_{x}+c_{1} \tilde{e}_{y}+d_{1} \tilde{e}_{z}=f_{1}, \\
\tilde{e}_{y}^{\prime \prime}+a_{2} \tilde{e}_{z}^{\prime}+b_{2} \tilde{e}_{x}+c_{2} \tilde{e}_{y}+d_{2} \tilde{e}_{z}=f_{2}, \\
\tilde{e}_{x}^{\prime}+a_{3} \tilde{e}_{y}^{\prime}+b_{3} \tilde{e}_{x}+c_{3} \tilde{e}_{y}+d_{3} \tilde{e}_{z}=f_{3},
\end{gathered}
$$

where: $a_{1}=-i k_{x}, b_{1}=k_{0}^{2} \varepsilon_{x x}-k_{y}^{2}, \quad c_{1}=k_{0}^{2} \varepsilon_{x y}+k_{x} k_{y}$,

$$
d_{1}=k_{0}^{2} \varepsilon_{x z}, \quad a_{2}=-i k_{y}, \quad b_{2}=k_{0}^{2} \varepsilon_{y x}+k_{x} k_{y},
$$$$
c_{2}=k_{0}^{2} \varepsilon_{y y}-k_{x}^{2}, \quad d_{2}=k_{0}^{2} \varepsilon_{y z}, \quad a_{3}=k_{y} / k_{x},
$$$$
b_{3}=i\left(k_{0}^{2} / k_{x}\right) \varepsilon_{z y}, c_{3}=i\left(k_{0}^{2} / k_{x}\right) \varepsilon_{x y},
$$$$
d_{3}=-i\left(k_{x}^{2}+k_{y}^{2}-k_{0}^{2} \varepsilon_{z z}\right) / k_{x}, \quad f_{1}=-k_{0}^{2} g_{x},
$$$$
f_{2}=-k_{0}^{2} g_{y}, \quad f_{3}=-i\left(k_{0}^{2} / k_{x}\right) g_{z}
$$

Let yz-plane coincides with the lower boundary of a slab. The boundary conditions are: at $z \geq L$ ( $L$ is a thickness of inhomogeneous plasma slab) waves propagating in negative direction must be absent, at $z \leq 0$ waves propagating in positive direction must be absent.

As far as all functions are finite inside a turbulent plasma slab, $0 \leq z \leq L$, we solve the set of equations (14) using the spectral method:

$$
\begin{aligned}
& {\left[\begin{array}{l}
\tilde{e}_{x}(z) \\
\tilde{e}_{y}(z) \\
\tilde{e}_{z}(z)
\end{array}\right]=\frac{1}{2 \pi} \int_{-\infty}^{\infty} d t\left[\begin{array}{l}
A(t) \\
B(t) \\
C(t)
\end{array}\right] \exp [-i(L-z) t],} \\
& {\left[\begin{array}{l}
f_{1}(z) \\
f_{2}(z) \\
f_{2}(z)
\end{array}\right]=\frac{1}{2 \pi} \int_{-\infty}^{\infty} d t\left[\begin{array}{l}
F_{1}(t) \\
F_{2}(t) \\
F_{3}(t)
\end{array}\right] \exp [-i(L-z) t] .}
\end{aligned}
$$

Set of stochastic differential equations (13) will be transformed into set of algebraic equations:

$$
\begin{aligned}
& \alpha_{1}(t) A(t)+c_{1} B(t)+\alpha_{2}(t) C(t)=F_{1}(t), \\
& b_{2} A(t)+\beta_{1}(t) B(t)+\beta_{2}(t) C(t)=F_{2}(t), \\
& \gamma_{1}(t) A(t)+\gamma_{2}(t) B(t)+d_{3} C(t)=F_{1}(t),
\end{aligned}
$$

where: $\alpha_{1}(t)=b_{1}-t^{2}, \alpha_{2}(t)=d_{1}+i a_{1} t, \beta_{1}(t)=c_{2}-t^{2}$, $\beta_{2}(t)=d_{2}+i a_{2} t, \gamma_{1}(t)=b_{3}+i t, \quad \gamma_{2}(t)=c_{3}+i a_{3} t$. Introducing the designation $x=t / k_{0}$ for the determinant of equations (16) we obtain:

$$
\Delta(x)=-\frac{i}{k_{x}} k_{0}^{6}\left(Q_{4} x^{4}+Q_{3} x^{3}+Q_{2} x^{2}+Q_{1} x+Q_{0}\right),
$$

where: $Q_{4}=2 \gamma^{2}-\varepsilon_{z z}, \quad Q_{2}=-\left[\left(\varepsilon_{x z}^{2}-\varepsilon_{y z}^{2}-\varepsilon_{x x} \varepsilon_{z z}-\right.\right.$ $\left.\left.-\varepsilon_{y y} \varepsilon_{z z}\right)+\gamma_{x}^{2}\left(2 \varepsilon_{x x}+\varepsilon_{y y}+\varepsilon_{z z}\right)\right], Q_{3}=2 \gamma_{y} \varepsilon_{y x}$, $Q_{1}=-\gamma_{y}\left[2\left(\varepsilon_{x y} \varepsilon_{z x}+\varepsilon_{x x} \varepsilon_{z y}\right)+\varepsilon_{y z}\left(2 \gamma_{x}^{2}-\gamma_{y}^{2}\right)\right]$, $Q_{0}=-\left[\left(\varepsilon_{x x} \varepsilon_{y y} \varepsilon_{z z}+2 \varepsilon_{y z} \varepsilon_{z x} \varepsilon_{x y}-\varepsilon_{y y} \varepsilon_{x z}^{2}+\varepsilon_{x x} \varepsilon_{y z}^{2}+\right.\right.$ $\left.+\varepsilon_{z z} \varepsilon_{x y}^{2}\right)-\gamma_{x}^{2}\left(\varepsilon_{x x} \varepsilon_{z z}+\varepsilon_{x x} \varepsilon_{y y}-\varepsilon_{x z}^{2}+\varepsilon_{x y}^{2}\right)-$ 
$\left.-\gamma_{y}^{2}\left(\varepsilon_{x x} \varepsilon_{y y}+\varepsilon_{y y} \varepsilon_{z z}+\varepsilon_{y z}^{2}+\varepsilon_{x y}^{2}\right)\right], \gamma_{x}=k_{x} / k_{0}$,

$\gamma_{y}=k_{y} / k_{0}, \gamma^{2}=\gamma_{x}^{2}+\gamma_{y}^{2}$.

Algebraic equation $\Delta(x)=0$ has four roots:

$$
\begin{aligned}
& x_{1,2}=\sqrt{\frac{\alpha_{0}}{2}} \pm\left[-\alpha_{0}-\frac{Q_{2}}{2 Q_{4}}+\frac{3 Q_{3}^{2}}{16 Q_{4}^{2}}-\right. \\
& \left.-\frac{\sqrt{2}\left(-4 Q_{2} Q_{3} Q_{4}^{2}+Q_{3}^{2} Q_{4}+8 Q_{1} Q_{4}^{3}\right)}{32 Q_{4}^{4} \sqrt{\alpha_{0}}}\right]-\frac{Q_{3}}{4 Q_{4}},
\end{aligned}
$$$$
x_{3,4}=-\sqrt{\frac{\alpha_{0}}{2}} \pm\left[-\alpha_{0}-\frac{Q_{2}}{2 Q_{4}}+\frac{3 Q_{3}^{2}}{16 Q_{4}^{2}}+\right.
$$$$
\left.+\frac{\sqrt{2}\left(-4 Q_{2} Q_{3} Q_{4}^{2}+Q_{3}^{2} Q_{4}+8 Q_{1} Q_{4}^{3}\right)}{32 Q_{4}^{4} \sqrt{\alpha_{0}}}\right]-\frac{Q_{3}}{4 Q_{4}},
$$

where $\alpha_{0}$ is any root of the third-order equation:

$$
\begin{aligned}
& \alpha^{3}+\frac{8 Q_{2} Q_{4}-3 Q_{3}^{2}}{8 Q_{4}^{2}} \alpha^{2}+\left[\frac{\left(8 Q_{2} Q_{4}-3 Q_{3}^{2}\right)^{2}}{256 Q_{4}^{4}}-\right. \\
& \left.-\frac{\left.64 Q_{1} Q_{3} Q_{4}^{2}+256 Q_{0} Q_{4}^{3}+16 Q_{2} Q_{3}^{2} Q_{4}-3 Q_{3}^{4}\right)}{256 Q_{4}^{4}}\right] \alpha- \\
& -\frac{\left(-4 Q_{2} Q_{3} Q_{4}+Q_{3}^{3}+8 Q_{1} Q_{4}^{2}\right)^{2}}{64 Q_{4}^{6}}=0,
\end{aligned}
$$

which can be solved by the Kardan-Ferrary method [13].

At quasi-longitudinal propagation determinant (17) has four roots [11]:

$$
\begin{aligned}
x_{1}=\zeta_{1}-\zeta_{2} \gamma^{2}, x_{2} & =\zeta_{3}-\zeta_{4} \gamma^{2}, \quad x_{3}=-x_{1}, \\
x_{4} & =-x_{2}
\end{aligned}
$$

where: $\zeta_{1}=\left(\varepsilon_{x x}+\tilde{\varepsilon}_{x y}\right)^{1 / 2}, \quad \zeta_{3}=\left(\varepsilon_{x x}-\tilde{\varepsilon}_{x y}\right)^{1 / 2}$,

$$
\zeta_{2}=\frac{1}{4 \varepsilon_{z z}} \frac{\varepsilon_{x x}+\varepsilon_{z z}+\tilde{\varepsilon}_{x y}}{\sqrt{\varepsilon_{x x}+\tilde{\varepsilon}_{x y}}}, \quad \zeta_{4}=\frac{1}{4 \varepsilon_{z z}} \frac{\varepsilon_{x x}+\varepsilon_{z z}-\tilde{\varepsilon}_{x y}}{\sqrt{\varepsilon_{x x}-\tilde{\varepsilon}_{x y}}} .
$$

These formulas were obtained at: $k_{0} l_{\|} \gg 1, \quad k_{0} L>>1$, $L>>l_{\|}$, where $l_{\|}$is the characteristic spatial scale of plasma irregularities.

Solving functions $A(x), B(x)$ and $C(x)$ we obtain components of scattered electric fields satisfying the boundary conditions:

$e_{x}(\mathfrak{x}, L)=-\frac{2 k_{0}}{\delta_{1} \varepsilon_{z z}}<E_{x}>Y_{0} \Upsilon_{1}\left(c_{1} \gamma_{x}^{2}+b_{1} \gamma_{y}^{2}+i a_{1} \gamma_{x} \gamma_{y}\right)$
$\int_{0}^{L} d z^{\prime} n_{1}\left(\mathfrak{x}, z^{\prime}\right) \sin \left[\left(L-z^{\prime}\right) k_{0} x_{1}\right]-\frac{2 k_{0}}{\delta_{2} \varepsilon_{z z}} \Psi_{0} \Upsilon_{1}<E_{x}>$ $\left(b_{2}+d_{2} \gamma_{x}^{2}+c_{2} \gamma_{y}^{2}+i a_{2} \gamma_{x} \gamma_{y}\right)$

$\int_{0}^{L} d z^{\prime} n_{1}\left(\mathbf{e}, z^{\prime}\right) \sin \left[\left(L-z^{\prime}\right) k_{0} x_{2}\right]$,

$e_{y}(\mathfrak{e}, L)=-\frac{2 k_{0}}{\delta_{1} \varepsilon_{z z}}<E_{x}>Y_{0} \Upsilon_{1}\left[a_{1} \gamma_{x} \gamma_{y}+i\left(b_{1} \gamma_{x}^{2}+c_{1} \gamma_{y}^{2}\right)\right]$

$\int_{0}^{L} d z^{\prime} n_{1}\left(\mathfrak{a}, z^{\prime}\right) \sin \left[\left(L-z^{\prime}\right) k_{0} x_{1}\right]-\frac{2 k_{0}}{\delta_{2} \varepsilon_{z z}} \Psi_{0} \Upsilon_{1}<E_{x}>$

$\left[a_{2} \gamma_{x} \gamma_{y}+i\left(b_{2}+c_{2} \gamma_{x}^{2}+d_{2} \gamma_{y}^{2}\right)\right]$

$\int_{0}^{L} d z^{\prime} n_{1}\left(\mathfrak{æ}, z^{\prime}\right) \sin \left[\left(L-z^{\prime}\right) k_{0} x_{2}\right]$,

where: $\mathfrak{x}=\left\{k_{x}, k_{y}\right\}$ is the transversal wave number, $a_{1}=\zeta_{1}^{2}-\varepsilon_{z z}, b_{1}=2 \zeta_{1} \zeta_{2} \varepsilon_{z z}-\varepsilon_{x x}-\tilde{\varepsilon}_{x y}, \delta_{1}=4 \zeta_{1} \tilde{\varepsilon}_{x y}$, $c_{1}=\left(2 \zeta_{1} \zeta_{2}-1\right) \varepsilon_{z z}, \quad a_{2}=\zeta_{3}^{2}-\varepsilon_{z z}, \quad b_{2}=2 \tilde{\varepsilon}_{x y} \varepsilon_{z z}$,

$c_{2}=2 \zeta_{3} \zeta_{4} \varepsilon_{z z}-\tilde{\varepsilon}_{x y}-\varepsilon_{x x}, \Upsilon_{1}=1+\sqrt{u_{0}}$, $d_{2}=2 \zeta_{3} \zeta_{4} \varepsilon_{z z}-2 \tilde{\varepsilon}_{x y}-\varepsilon_{z z}, \quad \delta_{2}=-4 \zeta_{3} \tilde{\varepsilon}_{x y}$.

Second-order statistical moment of scattered electric field in the $x y$-plane has the following form:

$<e_{x}\left(x+\rho_{x}, y+\rho_{y}, L\right) e_{x}^{*}(x, y, L)>=-4 \frac{b_{2} L Y_{0}^{2} \Upsilon_{1}^{2}}{\delta_{2} \varepsilon_{z z}^{2}}<E_{x}>^{2}$

$\int_{-\infty}^{\infty} d k_{x} \int_{-\infty}^{\infty} d k_{y} \exp \left(i k_{x} \rho_{x}+i k_{y} \rho_{y}\right) \int_{-\infty}^{\infty} d \rho_{z} W_{D}\left(k_{x}, k_{y}, \rho_{z}\right)$

$\left\{\frac{1}{\delta_{1}}\left(c_{1} k_{x}^{2}+b_{1} k_{y}^{2}\right)\left[\frac{\sin \left(y k_{0} L\right)}{y k_{0} L} \cos \left(\frac{t}{2} k_{0} \rho_{z}\right)-\right.\right.$

$\left.-\frac{\sin \left(t k_{0} L\right)}{t k_{0} L} \cos \left(\frac{y}{2} k_{0} \rho_{z}\right)\right]+\frac{i}{\delta_{1}} a_{1} k_{x} k_{y}$

$\left[\frac{1-\cos \left(y k_{0} L\right)}{y k_{0} L} \sin \left(\frac{t}{2} k_{0} \rho_{z}\right)-\frac{1-\cos \left(t k_{0} L\right)}{t k_{0} L} \sin \left(\frac{y}{2} k_{0} \rho_{z}\right)\right]$ $+\frac{1}{\delta_{2}}\left(b_{2} k_{0}^{2}+d_{2} k_{x}^{2}+c_{2} k_{y}^{2}\right)$

$\left.\left[\frac{\sin \left(2 x_{2} k_{0} L\right)}{2 x_{2} k_{0} L}-\cos \left(x_{2} k_{0} \rho_{z}\right)\right]\right\}$.

Cross-correlation function of scattered radiation in the $x y$ plane is: 


$$
\begin{aligned}
& <e_{x}\left(x+\rho_{x}, y+\rho_{y}, L\right) e_{y}^{*}(x, y, L)>_{D}=-2 \frac{b_{2} L Y_{0}^{2} \Upsilon_{1}^{2}}{\delta_{2} \varepsilon_{z z}^{2}} \\
& <E_{x}>^{2} \int_{-\infty}^{\infty} d k_{x} \int_{-\infty}^{\infty} d k_{y} \exp \left(i k_{x} \rho_{x}+i k_{y} \rho_{y}\right) \int_{-\infty}^{\infty} d \rho_{z} \\
& W_{D}\left(k_{x}, k_{y}, \rho_{z}\right)\left\{\frac{1}{\delta_{1}}\left[2 a_{1} k_{x} k_{y}-i\left(c_{1}+b_{1}\right)\left(k_{x}^{2}+k_{y}^{2}\right)\right]\right. \\
& {\left[\frac{\sin \left(y k_{0} L\right)}{y k_{0} L} \cos \left(\frac{t}{2} k_{0} \rho_{z}\right)-\frac{\sin \left(t k_{0} L\right)}{t k_{0} L} \cos \left(\frac{y}{2} k_{0} \rho_{z}\right)\right]} \\
& -\frac{i}{\delta_{1}}\left(c_{1}-b_{1}\right)\left(k_{x}^{2}-k_{y}^{2}\right)\left[\frac{1-\cos \left(y k_{0} L\right)}{y k_{0} L} \sin \left(\frac{t}{2} k_{0} \rho_{z}\right)\right. \\
& \left.-\frac{1-\cos \left(t k_{0} L\right)}{t k_{0} L} \sin \left(\frac{y}{2} k_{0} \rho_{z}\right)\right]+\frac{1}{\delta_{2}}\left[-2 i b_{2} k_{0}^{2}+\right. \\
& \left.+2 a_{2} k_{x} k_{y}-i\left(d_{2}+c_{2}\right)\left(k_{x}^{2}+k_{y}^{2}\right)\right] \\
& \left.\left[\frac{\sin \left(2 x_{2} k_{0} L\right)}{2 x_{2} k_{0} L}-\cos \left(x_{2} k_{0} \rho_{z}\right)\right]\right\},
\end{aligned}
$$

where: $\rho_{y}$ and $\rho_{x}$ are distances between observation points spaced apart in the principle and perpendicular planes, respectively, $W_{D}\left(k_{x}, k_{y}, \rho_{z}\right)$ is the arbitrary two-dimensional spectral function of electron density fluctuations. These expressions are valid for the near $(R<<1)$ and far $(R>>1)$ zones from plasma slab boundaries, $R=L / k_{0} l_{\|}^{2}$ is the wavy parameter.

\section{Polarimetric parameters}

Knowledge of the correlation functions of scattered fields allows to calculate Stokes parameters

$$
\begin{gathered}
I=<e_{x} e_{x}^{*}>+<e_{y} e_{y}^{*}>, \quad Q=<e_{x} e_{x}^{*}>-<e_{y} e_{y}^{*}>, \\
U=2 \operatorname{Re}\left(<e_{x} e_{y}^{*}>\right), \quad V=2 \operatorname{Im}\left(<e_{x} e_{y}^{*}>\right) .
\end{gathered}
$$

In general the set of parameters (26) describes elliptically polarized wave. Depolarization degree is the ratio of the unpolarized component energy to the energy of the wave

$$
\Xi=\frac{I-\left(Q^{2}+U^{2}+V^{2}\right)^{1 / 2}}{I} .
$$

For a completely polarized wave, $I^{2}=Q^{2}+U^{2}+V^{2}$, and polarization fluctuations are absent.

Still more interesting than the Stokes parameters are the following three measures. The total polarization $P$, the degree of ellipticity $E$ and the degree of linear polarization $L$ given by:

$$
\begin{gathered}
P=\frac{\left(Q^{2}+U^{2}+V^{2}\right)^{1 / 2}}{I}, \quad E=\frac{V}{\left(Q^{2}+U^{2}+V^{2}\right)^{1 / 2}} \\
L=\frac{\left(Q^{2}+U^{2}\right)^{1 / 2}}{\left(Q^{2}+U^{2}+V^{2}\right)^{1 / 2}}
\end{gathered}
$$

For elliptic polarized wave $P=1$, for partially polarization $0<P<1$ and for unpolarized wave $P=0$. These parameters are of interest having wide application because they are invariant under Lorentz transformation and may be used in astrophysical applications, which is not true of the Stokes parameters.

Using statistical characteristics of scattered electromagnetic waves Stokes parameters can be easily calculated:

$$
\begin{aligned}
& I=-4 \frac{b_{2} L Y_{0}^{2} \Upsilon_{1}^{2}}{\delta_{2} \varepsilon_{z z}^{2}}<E_{x}>^{2} \int_{-\infty}^{\infty} d k_{x} \int_{-\infty}^{\infty} d k_{y} \\
& \exp \left(i k_{x} \rho_{x}+i k_{y} \rho_{y}\right) \int_{-\infty}^{\infty} d \rho_{z} W_{D}\left(k_{x}, k_{y}, \rho_{z}\right) \\
& \left\{\frac { 1 } { \delta _ { 1 } } ( b _ { 1 } + c _ { 1 } ) ( k _ { x } ^ { 2 } + k _ { y } ^ { 2 } ) \left[\frac{\sin \left(y k_{0} L\right)}{y k_{0} L} \cos \left(\frac{t}{2} k_{0} \rho_{z}\right)-\right.\right.
\end{aligned}
$$

$$
\begin{aligned}
& \left.-\frac{\sin \left(t k_{0} L\right)}{t k_{0} L} \cos \left(\frac{y}{2} k_{0} \rho_{z}\right)\right]+\frac{1}{\delta_{2}}\left[2 b_{2} k_{0}^{2}+\left(c_{2}+d_{2}\right)\right. \\
& \left.\left.\left(k_{x}^{2}+k_{y}^{2}\right)\right]\left[\frac{\sin \left(2 x_{2} k_{0} L\right)}{2 x_{2} k_{0} L}-\cos \left(x_{2} k_{0} \rho_{z}\right)\right]\right\},
\end{aligned}
$$

$Q=-4 \frac{b_{2} L Y_{0}^{2} \Upsilon_{1}^{2}}{\delta_{2} \varepsilon_{z z}^{2}}<E_{x}>^{2} \int_{-\infty}^{\infty} d k_{x} \int_{-\infty}^{\infty} d k_{y}$

$\exp \left(i k_{x} \rho_{x}+i k_{y} \rho_{y}\right) \int_{-\infty}^{\infty} d \rho_{z} W_{D}\left(k_{x}, k_{y}, \rho_{z}\right)\left\{\frac{1}{\delta_{1}}\left(c_{1}-b_{1}\right)\right.$

$$
\left(k_{x}^{2}-k_{y}^{2}\right)\left[\frac{\sin \left(y k_{0} L\right)}{y k_{0} L} \cos \left(\frac{t}{2} k_{0} \rho_{z}\right)-\frac{\sin \left(t k_{0} L\right)}{t k_{0} L}\right.
$$

$\left.\cos \left(\frac{y}{2} k_{0} \rho_{z}\right)\right]+i \frac{2}{\delta_{1}} a_{1} k_{x} k_{y}\left[\frac{1-\cos \left(y k_{0} L\right)}{y k_{0} L}\right.$

$$
\left.\sin \left(\frac{t}{2} k_{0} \rho_{z}\right)-\frac{1-\cos \left(t k_{0} L\right)}{t k_{0} L} \sin \left(\frac{y}{2} k_{0} \rho_{z}\right)\right]+\frac{1}{\delta_{2}}\left(d_{2}-\right.
$$

$\left.\left.-c_{2}\right)\left(k_{x}^{2}-k_{y}^{2}\right)\left[\frac{\sin \left(2 x_{2} k_{0} L\right)}{2 x_{2} k_{0} L}-\cos \left(x_{2} k_{0} \rho_{z}\right)\right]\right\}$. 


\section{Numerical calculations}

The incident electromagnetic wave having frequency of 3 $\mathrm{MHz}\left(k_{0}=6.28 \cdot 10^{-2} \mathrm{~m}^{-1}\right)$ propagates along the $Z$-axis. Plasma parameters at the altitude of $300 \mathrm{~km}$ are: $u_{0}=0.22$, $\mathrm{v}_{0}=0.28$. The first Fresnel radius and the Fresnel wavenumber are equal to $5.5 \mathrm{~km}$ and $0.64 \mathrm{~km}^{-1}$, respectively.

In the experiment [14] which used transmission through the ionosphere from satellite beacons, were shown that nighttime irregularities are aligned along the earth's magnetic field and appear to extend from top to bottom of the F-region. They are much more elongated than those found for most of the spaced receiver drift measurements. The irregularities are observable structures as small as a few hundred meters. Small scale $(<200 \mathrm{~m})$ ionospheric irregularities with the Gaussian spectrum are responsible for polarization fluctuations [15].

Thermal plasma density measurements obtained by using the spherical electrostatic analyzer probe carried by the polar orbiting satellite show have been shown [16] that the range of scale sizes examined, $200 \mathrm{~m}$ to over $100 \mathrm{~km}$ under normal conditions. Irregularities in the high-latitude region have power spectrum the index within the range 1.5-2.5. The most important dynamic and statistical back scattered signal (BSS) characteristics were measured [17] using probe waves in the frequency range of $1.68-6 \mathrm{MHz}$ that made it possible to obtain the basic parameters of the plasma irregularities. During the experiments the BSS amplitudes and some important dynamic and statistical characteristics were measured. The space distance antenna reception of BSS allowed to find out the anisotropy character of the scattered field. Theirs directions changed. The spectral index was equal to $p=1.4 \div 4.8$ in different heating sessions with the average $\langle p\rangle=3.05$ in the probe wave frequency range of $2.6 \div 6 \mathrm{MHz}$.

Data obtained from spaced receiver measurements made at Kingston, Jamaica (during the periods August 1967January 1969 and June 1970-September 1970) show that the irregularities between heights of 153 and $617 \mathrm{~km}$ causing the scintillation of signals from the moving earth satellites (BE-B and $\mathrm{BE}-\mathrm{C}$ ) are closely aligned along the magnetic field lines in the $F$-region [18]. Orientation of the irregularities in the ionosphere has been measured with respect to the geographic north observing a diffraction pattern of the satellite signals (41 $\mathrm{MHz}$ ) on the ground. The dip angle of the irregularities with respect to the field lines was within $16^{0}$. The anisotropic spectral features in the $F$-region are defined for Gaussian and Power-law spectra. Average value of the variance of electron density fluctuations $\sigma_{n}^{2}=<n_{1}^{2}>/ N_{0}^{2}$ at the frequencies of 25 MHz and $30 \mathrm{MHz}$ is in the range $\sigma_{N} \simeq 10^{-3} \div 10^{-5}$.

We will use new spectrum of electron density irregularities combining anisotropic Gaussian and power-law spectra [4]:

$$
W_{n}(\mathbf{k})=\frac{\sigma_{n}^{2}}{8 \pi^{5 / 2}} \frac{A_{p} l_{\|}^{3}}{\chi^{2}\left[1+l_{\perp}^{2}\left(k_{x}^{2}+k_{y}^{2}\right)+l_{\|}^{2} k_{z}^{2}\right]^{p / 2}}
$$

$\exp \left(-\frac{k_{x}^{2} l_{\perp}^{2}}{4}-p_{1} \frac{k_{y}^{2} l_{\|}^{2}}{4}-p_{2} \frac{k_{z}^{2} l_{\|}^{2}}{4}+p_{3} k_{y} k_{z} l_{\|}^{2}\right)$,

where: $p_{1}=\left(\sin ^{2} \gamma_{0}+\chi^{2} \cos ^{2} \gamma_{0}\right)^{-1}\left[1+\left(\chi^{2}-1\right)^{2}\right.$

$\left.\sin ^{2} \gamma_{0} \cos ^{2} \gamma_{0} / \chi^{2}\right], p_{2}=\left(\sin ^{2} \gamma_{0}+\chi^{2} \cos ^{2} \gamma_{0}\right) / \chi^{2}$,

$p_{3}=\left(\chi^{2}-1\right) \sin \gamma_{0} \cos \gamma_{0} / 2 \chi^{2}, A_{p}=\Gamma(p / 2)$

$\Gamma[(5-p) / 2] \sin [(p-3) \pi / 2], k_{x}, k_{y}$ and $k_{z}$ are the wave vector $\mathbf{k}$ components perpendicular $\left(k_{x}, k_{y}\right)$ and parallel $\left(k_{\|}\right)$to the incident wave propagation, $\chi=l_{\|} / l_{\perp}$ is the anisotropy factor - the ratio of longitudinal and transverse characteristic linear sizes of plasma irregularities, $\gamma_{0}$ is the orientation angle of elongated ionospheric plasma irregularities with respect to the magnetic lines of force. The shape of electron density irregularities has a spheroidal form. Anisotropy of the shape of irregularities is connected with the difference of the diffusion coefficients in the field align and field perpendicular directions.

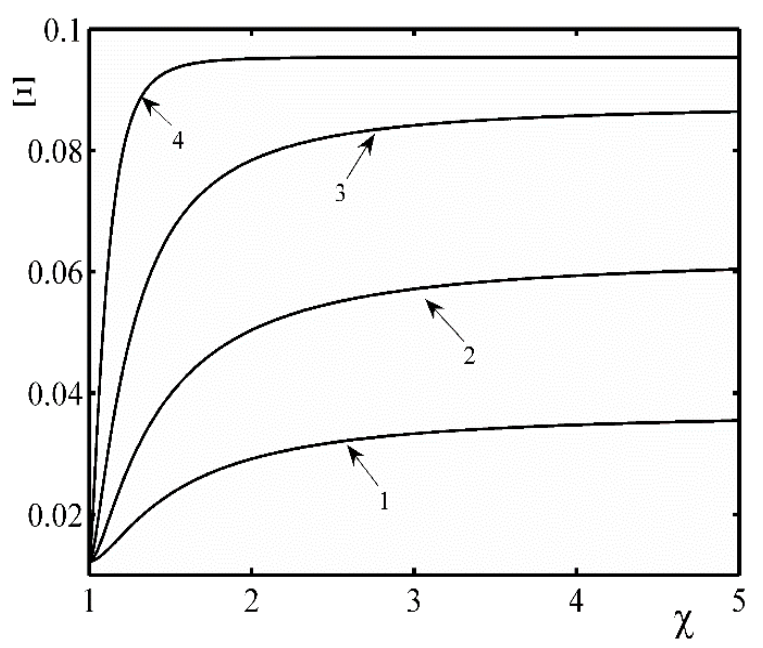

Figure 1: Normalized depolarization degree $\Xi$ anisotropy factor $\chi$; curve 1 corresponds to $\gamma_{0}=0.5^{0}$, curve $2-\gamma_{0}=1^{0}$, curve $3-\gamma_{0}=2^{0}$, curve $4-\gamma_{0}=5^{0}$.

Numerical calculations are carried out for characteristic linear scale of small-scale ionospheric irregularities is $80 \mathrm{~m}-$ $200 \mathrm{~m}$, thickness of a slab is $40 \mathrm{~km}$.

Figure 1 depicts $\Xi$ depolarization degree of scattered electromagnetic waves versus anisotropy factor for different inclination angle $\gamma_{0}=0.5^{0}-5^{0}$ for small-scale plasma irregularities $l_{\|}=160 \mathrm{~m}$. Parameter $\Xi$ increasing in proportion of the orientation angle of elongated irregularities with respect to the direction of the earth's magnetic lines of force. Two observation points are spaced apart normal to the main plane $640 \mathrm{~m}$. 


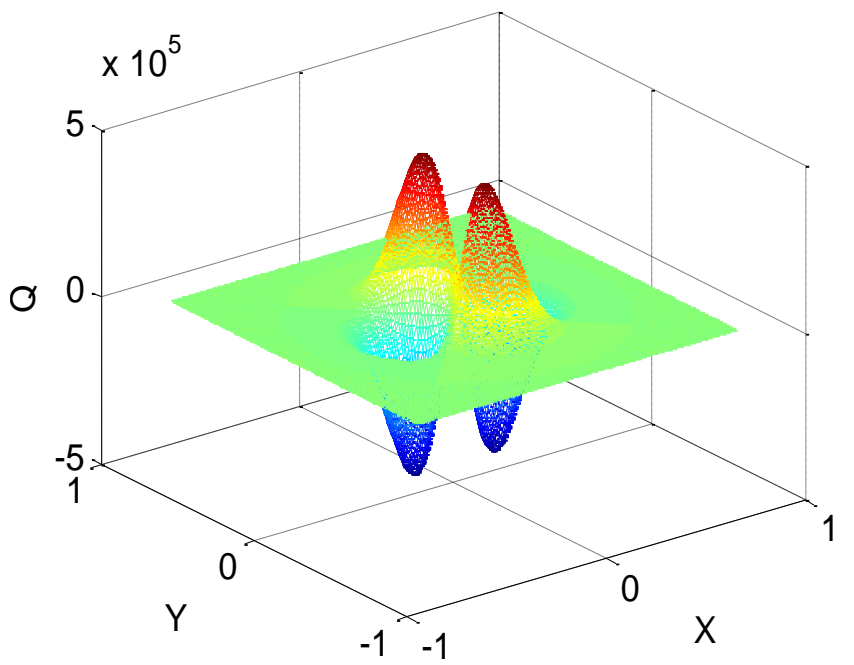

Figure 2: The second Stokes parameter versus distances between observation points.

Numerical calculations show that first Stokes parameter $(I)$ representing the total radiated power density has maximum at the anisotropy factor $\chi=2$. Intensity of scattered radiation decreases in proportion to the parameter $\chi$ and fading nulls. Figure 2 illustrates 3D picture of the second Stokes parameter $(Q)$ representing the degree of polarization. This parameter can be both positive and negative values and this is the important parameter in the scintillation effect. Particularly, for a field aligned small scale irregularities it is positive having maximum at $\chi=2$, if $\eta_{x}=1$ and $\eta_{y}=0$; negative if $\eta_{x}=0$ and $\eta_{y}=1$ (here $\eta_{x, y}=\rho_{x, y} / l_{\|}$is the nondimensional parameter - distances between observation points are normalized on the linear scale of plasma irregularities).

For $3 \mathrm{MHz}$ and $40 \mathrm{MHz}$ incident wave scattered electromagnetic waves are elliptic polarized $(P=1)$ for field aligned irregularities $\left(\gamma_{0}=0^{0}\right)$. At the angle $\gamma_{0}=10^{0}$, $\chi=5$ and $\chi=10$ scattered radiation is partially polarized $P=0.79(0<P<1)$ for $3 \mathrm{MHz}$ incident wave; increasing frequency up to $40 \mathrm{MHz}, P=0.24$ at $\chi=5$ and $P=0.22$ at $\chi=10$. That is orientation angle and anisotropy factor have an influence on the polarization of scattered electromagnetic waves.

Figure 3 illustrates the dependence of the degree of ellipticity $E$ versus anisotropy factor of ionospheric irregularities. Ellipticity decreases in proportion of the inclination angle $\gamma_{0}$ and tends to the saturation increasing parameter $\chi$. Saturation of the curves started at $\chi=6$ for $\gamma_{0}=0.05^{\circ}$ (irregularities approximately are field aligned) and at $\chi=1.5$ for $\gamma_{0}=2^{0}$. The degree of linear polarization $\mathrm{L}$ versus anisotropy factor is depicted in Figure 4. Linear polarization increases in proportion of the orientation angle of stretched ionospheric irregularities with respect to the external magnetic field, increasing anisotropy factor the curves tend to the saturation: curve 1 at $\chi=5$ curve 4 at $\chi=2$.

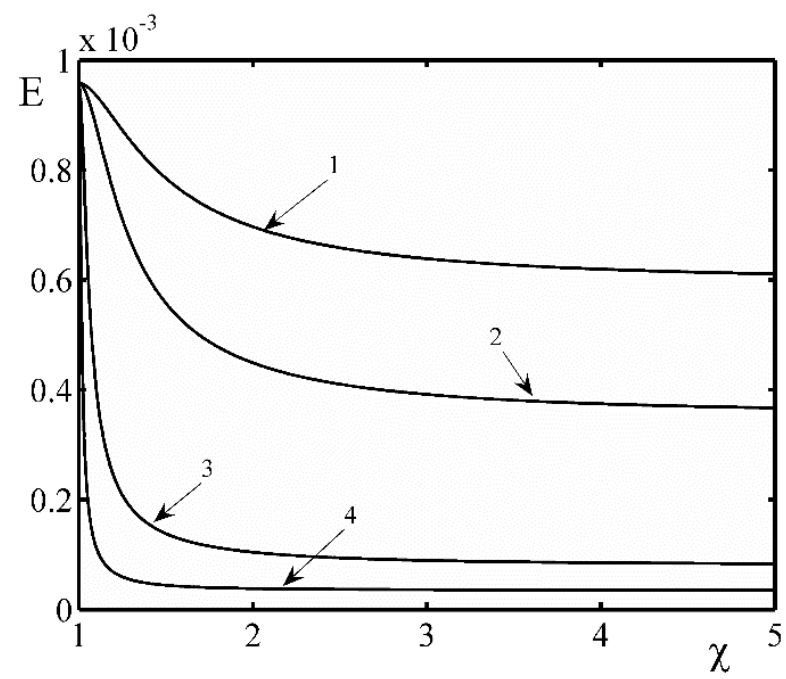

Figure 3: The degree of ellipticity E versus anisotropy factor $\chi$. curve 1 corresponds to $\gamma_{0}=0.05^{0}$, curve 2 - $\gamma_{0}=0.1^{0}$, curve $3-\gamma_{0}=0.5^{0}$, curve $4-\gamma_{0}=2^{0}$.

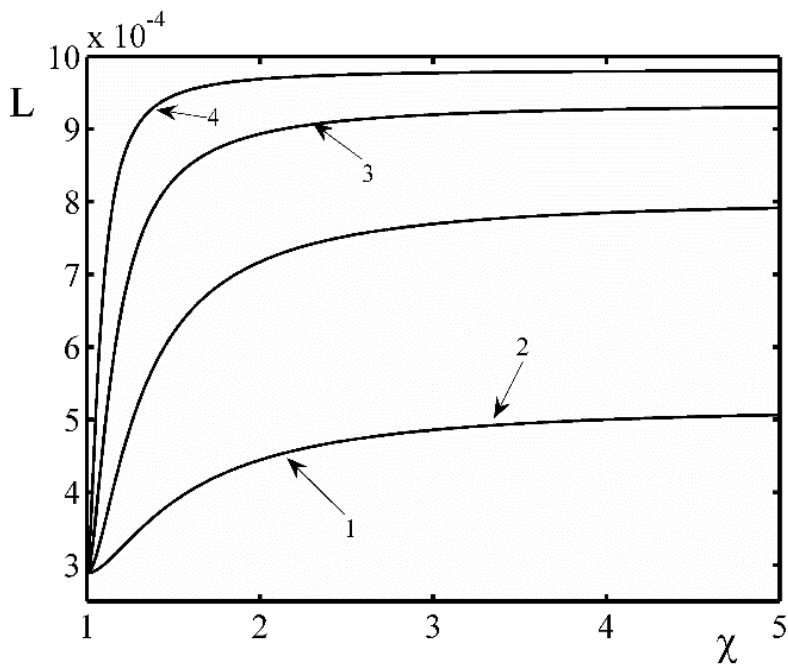

Figure 4: The degree of linear polarization $\mathrm{L}$ versus anisotropy factor $\chi$. Curve 1 corresponds to $\gamma_{0}=0.02^{0}$, curve $2-\gamma_{0}=0.05^{0}$, curve 3 $\gamma_{0}=0.1^{0}$, curve $4-\gamma_{0}=0.2^{0}$.

The depolarization effect depends on the strength of the random variations of the medium and is expected to be very sensitive to frequency. Application of the Stokes parameters at radio waves scattering in the upper ionosphere allows to determine characteristic spatial scales and anisotropy factors of irregularities solving the reverse problem.

\section{Conclusions}

The problem of wave propagation through a medium containing random irregularities is studied with the effect of 
anisotropic background on the fluctuations taken into account. The general formulation of the problem of how a slab of random medium affects the propagation and polarization of an incident electromagnetic wave is obtained. The solution of wave equation is given in Fourier series. It was shown that anisotropy factor of small-scale plasma irregularities has an influence on the polarimetric parameters of scattered radiation, while the role of the inclination angle is negligible. The obtained results are valid for both near and far zones from plasma a slab boundaries. The relationship between the scintillation effects and the polarimetric parameters is important. It was suggested that the second Stokes parameter may play important role in the scintillations as the small-scale plasma irregularities having metric characteristic linear scales should have sufficient scattering power. The depolarization effect may be appreciable for strong scintillation. The obtained Stokes parameters in the far zone from plasma layer boundaries allows to determine polarization parameters of scattered radio waves in the ionospheric $F$ region with great accuracy and also make the assumption of region in formation of depolarization of scattered radio waves. The theory should be generalized for conductive anisotropic turbulent media using the same approach as given in this paper.

\section{Acknowledgements}

This work was partially supported by the Ministry of Education, Youth and Sports: by the National Program of Sustainability (NPU II) project „IT4 Innovations excellence in science - LQ1602“.

\section{References}

[1] A. Ishimaru, Wave Propagation and Scattering in Random Media, Vol. 2, Multiple Scattering, Turbulence, Rough Surfaces and Remote Sensing, IEEE Press, Piscataway, New Jersey, 1997.

[2] G. Jandieri, A. Ishimaru, B. Rawat, V. Gavrilenko, O. Kharshiladze, Statistical moments and scintillation level of scattered electromagnetic waves in the magnetized plasma, PIER C 84: 11-22, 2018.

[3] G. Jandieri, Zh. Diasamidze, M. Diasamidze, Scintillation effects and the spatial power spectrum of scattered radio waves in the ionospheric $\mathrm{F}$ region, J. Advances Physics 13: 4593-4604, 2017.

[4] G. Jandieri, A. Ishimaru, B. Rawat, Zh. Diasamidze, Power spectra of ionospheric scintillations, Advanced Electromagnetics 6: 42-51, 2017.

[5] J.W. Strohbehn, S.F. Clifford, Polarization and angle-ofarrival fluctuations for a plane wave propagated through a turbulent medium, IEEE Trans. Antennas and Propagation, 15: 416-421, 1967.
[6] V.G. Gavrilenko, N.S. Stepanov, Depolarization of electromagnetic waves in turbulent cosmic plasma, Astronomic Journal 53: 291- 294, 1976.

[7] G. Jandieri, A. Ishimaru, V. Jandieri, Depolarization effects of incoherently scattered electromagnetic waves by inhomogeneous magnetized plasma slab, $J$. Electromagnetic Analysis and Application 3: 471-478, 2011.

[8] G. Jandieri, A. Ishimaru, V. Jandieri, Depolarization of metric radio signals and the spatial spectrum of scattered radiation by magnetized turbulent plasma slab, PIER 112: 63-75, 2011.

[9] R. Bourret, The depolarization of light by interstellar magnetic turbulence, Astrophysics and Space Sciences 24: 407-419, 1973

[10]R. Bourret, The depolarization of electromagnetic radiation in a random medium: evolution of the Stokes parameters, Optica Acta 21: 721-735, 1974.

[11] G.V. Jandieri, A. Ishimaru, , M.R. Diasamidze, On the influence of fluctuations of the direction of an external magnetic field on phase and amplitude correlation functions of scattered radiation by magnetized slab, PIER B 22: 121-143, 2010.

[12] V.L. Ginzburg, Propagation of Electromagnetic Waves in Plasma. New York: Gordon and Beach, 1961.

[13] G.A. Korn, J.M. Korn, Mathematical handbook for scientists and engineers. Definition, theorems and formulas for reference and review (McGraw Hill Book Company, New York, Toronto, London, Sydney, 1968.

[14] J.W. MacDougall, Distributions of the irregularities which produce ionospheric scintillations, J. Atmos. Terr. Phys. 43: 317-325, 1981.

[15]B.N. Gershman, L.M. Erukhimov, Yu.Ya. Yashin, Wave phenomena in the Ionosphere and Space Plasma, Moscow, Nauka, 1984.

[16] A.D.R. Phelps, R.C. Sagalyn, Plasma density irregularities in the high-latitude top side ionosphere, $J$. Geophys. Res. 81: 515-523, 1976.

[17] N.V. Bakhmet'eva, V.N. Bubukina, Yu.A. Ignat'ev, G.S. Bochkarev, V.A. Eremenko, V.V. Kol'sov, I.V. Kracheninnikov, Yu.N. Cherkashin, Investigation by backscatter radar of artificial irregularities produced in ionospheric plasma heating experiment, J. Atmos. Solar Terr. Phys. 59: 2257-2263, 1997.

[18] A.A. Chen, G.S. Kent, Determination of the orientation of ionospheric irregularities causing scintillation of signals from earth satellites, J. Atmos. Terr. Phys. 34: 1411-1414, 1972. 